\title{
Designed drug-release systems having various breathable polyurethane film-backed hydrocolloid acrylated adhesive layers for moisture healing
}

\author{
Ching-Hsien Chang ${ }^{\mathrm{a}, \mathrm{b}}$, Hsia-Wei Liu ${ }^{\mathrm{c}}$ and Ching-Cheng Huang ${ }^{\mathrm{d}, \mathrm{e},{ }^{*}}$ \\ ${ }^{a}$ Department of Chinese Medicine, En Chu Kong Hospital, New Taipei City, Taiwan \\ ${ }^{b}$ Department of Cosmetic Science, Chang Gung University of Science and Technology, Taoyuan, \\ Taiwan \\ ${ }^{c}$ Department of Life Science, Fu Jen Catholic University, New Taipei City, Taiwan \\ ${ }^{d}$ Department of Biomedical Engineering, Ming-Chuan University, Taoyuan, Taiwan \\ ${ }^{e}$ Regional R\&D Service Department, Metal Industries Research \& Development Centre, Taipei, \\ Taiwan
}

\begin{abstract}
A series of designed drug-release systems were prepared and established for clear moisture healing. These systems were designed to have an interpenetrating polymer network (IPN) structure, which contained a breathable polyurethane film, hydrocolloidlayer, and polyacrylate adhesive layer. Breathable polyurethane film $\left(2000 \mathrm{~g} / \mathrm{m}^{2} / 24 \mathrm{hr}\right)$ with high moisture permeability was employed as a base for new drug-release systems or wound dressings. All drug-release systems having a polyurethane film-backed hydrocolloid acrylated adhesive layer showed an increase of water uptakes with increasing time. After 114 hours, high water uptakes of drug-release systems with $20 \%$ hydrocolloid components were observed in the values of 160,1100 , and $1870 \%$ for different additional hydrocolloid components of carboxymethylcellulose, sodium alginate, and carbomer U10, respectively. New drug-release systems of polyurethane film-backed hydrocolloid/adhesive layers could be designed and established for wound care managements.
\end{abstract}

Keywords: Hydrocolloid, drug-release systems, breathable, carbomer

\section{Introduction}

Rapid and proper healing is important in the treatment of all wounds, including severe burns, trauma, diabetic and venous stasis ulcers, and similar tissue damages. The healing response of tissues involves a complex interaction between cells, extracellular matrix molecules, and soluble mediators. The complexity of this process has been simplified by dividing the healing response into four broad categories

${ }^{*}$ Corresponding author: Ching-Cheng Huang, Department of Biomedical Engineering, Ming-Chuan University, 5 De Ming Rd., Gui Shan District, Taoyuan, 333, Taiwan. Tel.: +886-988-915-063; Fax: +886-3-359-3840; E-mail: jcchuang@mail.mirdc.org.tw. 
that coincide with the temporal sequence of normal healing: homeostasis, inflammation, proliferation, and remodeling [1]. In cases of severe and significant skin loss or in the presence of difficult nonhealing wounds, immediate coverage of the wound surface with a dressing is needed. The dressing acts as natural skin by protecting the area from fluid and protein loss and preventing bacterial infection, and other subsequent tissue damage. Numerous skin replacements are either currently available for clinical use or are in clinical testing. These include temporary and permanent skin replacements, epidermal and dermal skin replacements, and synthetic/biosynthetic and biologic skin replacements. In this paper, we explore the design, synthesis, and preparation of new functional materials in order to determine which are suitable for biomedical application. These included polyurethane [2], polyacrylate [3,4], poltyamide [5], poltyimide [5], polyester [6], polynorborene [7-11], polydiphenylacetylenes [12], polytetrafluoroethylene, and polymeric resins [13-21]. Surface modification technology was also considered as a mode to change the superficial microenvironment of these materials [22-25].

\subsection{Materials}

Sodium alginate (NaAL) (purchased from Zhengzhou Sigma Chemical Co., Ltd., China), carbomer U10 (purchased from Zhengzhou Sigma Chemical Co., Ltd., China), carboxymethylcellulose (CMC) (purchased from Sigma, USA), and acrylate-base adhesives (purchased from Chensin Packing Industry Co. Ltd., Taiwan) were employed in this study. The chemical structures of sodium alginate (NaAL), carbomer U10, and carboxymethylcellulose (CMC) are shown in Scheme 1.

\subsection{Preparation of designed drug-release system with hydrocolloid acrylated adhesive layer}

A series of hydrocolloids were mixed with acrylate-base adhesives (purchased from Chensin Packing Industry Co. Ltd., Taiwan). This mixture was then coated onto select polymeric films, such as a polyurethane film with high moisture permeability $\left(2000 \mathrm{~g} / \mathrm{m}^{2} / 24 \mathrm{hr}\right)$. A series of designed drugrelease systems with an interpenetrating polymer network (IPN) structure were obtained. In the absence of a drug, this new drug-release system could be used as wound dressings for clear moisture healing [1].

\subsection{Water absorption of designed drug-release system with a hydrocolloid acrylated adhesive layer}

Various polymeric materials will absorb water when exposed. This may cause dimensional instability with property degradation, ultimately leading to failure. Depending on the application of the product, however, certain levels of moisture are acceptable. Certain materials require small amounts of moisture for ultimate field performance.

Absorption was used to evaluate materials by exposing specimens (2" Disks x $1 / 8$ " thick) to water for different lengths of time. The water absorption test was carried out by following ASTM D570.

(A)

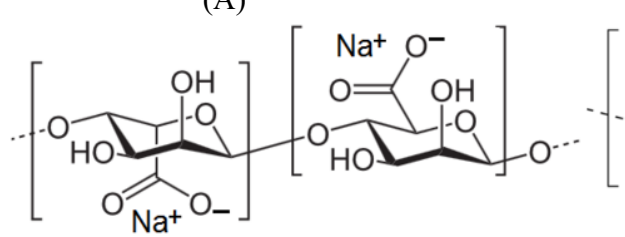

(B)

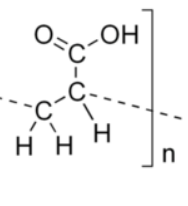

(C)

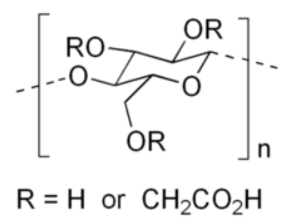

Scheme 1. The chemical structures of (A) sodium alginate (NaAL), (B) carbomer U10 and (C) carboxymethylcellulose (CMC). 


\section{Results and discussion}

2.1. Novel designed drug-release systems with interpenetrating polymer network having breathable polyurethane film-backed hydrocolloid acrylated adhesive layers

In cases where pharmaceuticals are not being administered, the new drug-release system could be used as a wound dressing for clear moisture healing [1]. Several important findings point to the efficacy of moist wound healing [1,26-28]. For example, cells proliferate and migrate faster in a moist healing environment such that the healing process is two times faster than it is in a completely dry environment. Epithelial cells proliferate and migrate faster on a moist non-clotting wound bed [1].

In clinical cases, dressings play an important role in the wound healing process. The moist healing process could reduce the length of healing time, formation of scars, and pain. Dehydration causes neurons to generate more pain signals in a dry healing environment of wound bed [1]. Oppositely, the exposed peripheral neurons in a moist healing environment of wound bed would generate fewer pain signals such that patients feel more comfortable during the healing process [1,26-28].

In this study, the designed drug-release system's breathable polyurethane film could be employed to form occlusive environments around a wound. The hydrocolloid acrylated adhesive layers would be swollen with tissue fluids for the preparation of a moist wound bed (see Figure 1) [1]. A polyurethane film with high moisture permeability $\left(2000 \mathrm{~g} / \mathrm{m}^{2} / 24 \mathrm{hr}\right)$ was employed in this study. As shown in Figure 1, the interpenetrating polymer network (IPN) structure would be swollen with tissue fluids. The shape of interpenetrating polymer network would be limited.

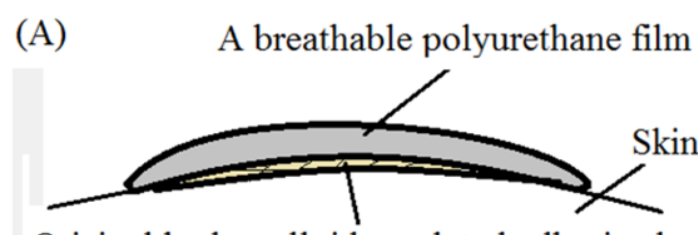

Original hydrocolloid acrylated adhesive layers without tissue fluids

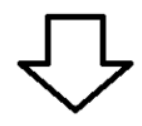

(B)

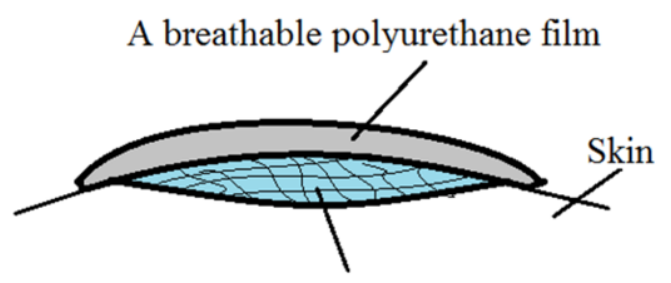

Swollen hydrocolloid acrylated adhesive layers with tissue fluids

Fig. 1. Proposed model of novel designed drug-release systems with an interpenetrating polymer network having breathable polyurethane film-backed hydrocolloid acrylated adhesive layers for moist wound healing. 


\subsection{Water absorption of designed drug-release system with a hydrocolloid acrylated adhesive layer}

A polyurethane film with high moisture permeability was employed as a base for drug-release systems. Various polymeric materials are susceptible to water absorption during exposure. This may cause dimensional instability with property degradation and may ultimately lead to failure. In this study, water absorption was employed to evaluate materials by exposing specimens to water. All drugrelease systems having a polyurethane (PU) film-backed hydrocolloid acrylated adhesive layer showed an increase of water uptake with increasing time in the initial stage. After 40 hours, high water uptakes of drug-release systems with $20 \%$ hydrocolloid components were observed in the values of $125 \%$, $700 \%$, and $880 \%$ for different additions of hydrocolloid components of carboxymethylcellulose $(\mathrm{CMC})$, sodium alginate (NaAL) and carbomer (U10), respectively (Figures 2-4). For some clinical applications, water uptake is important for long-term drug-release systems. After 114 hours, relatively high water uptakes of drug-release systems with $20 \%$ hydrocolloid components were observed in the values of $160 \%, 1100 \%$ and $1870 \%$ for different additional hydrocolloid components of carboxymethylcellulose (CMC), sodium alginate, and carbomer U10, respectively.

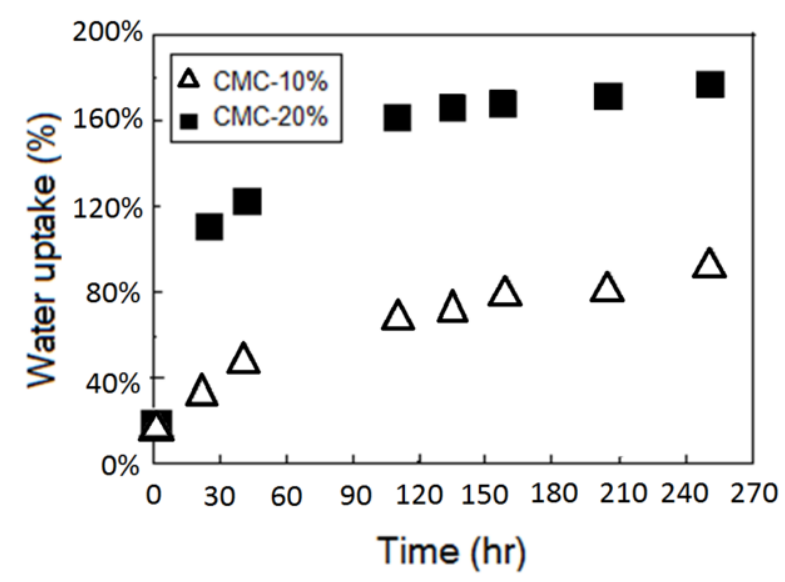

Fig. 2. Water uptakes of new designed drug-release system having a breathable polyurethane film-backed carboxymethylcellulose (CMC) hydrocolloidacrylated adhesive layer.

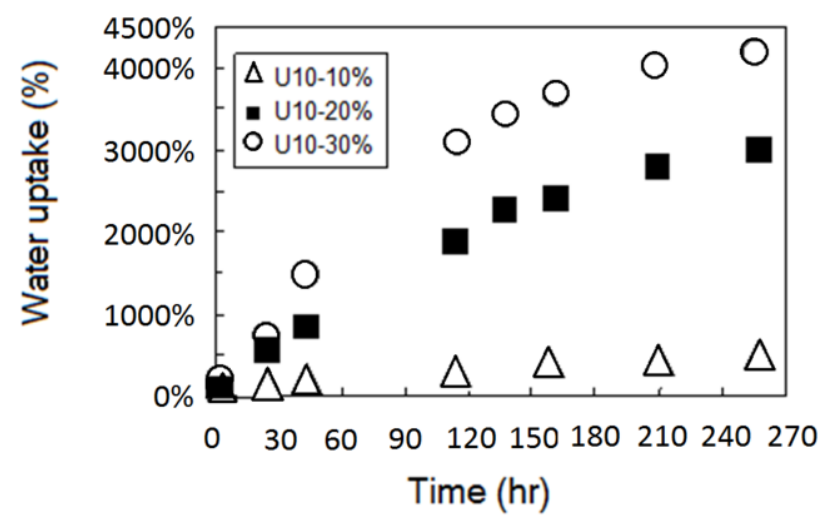

Fig. 3. Water uptakes of new designed drug-release system having a breathable polyurethane film-backed carbomer U10 hydrocolloidacrylated adhesive layer. 


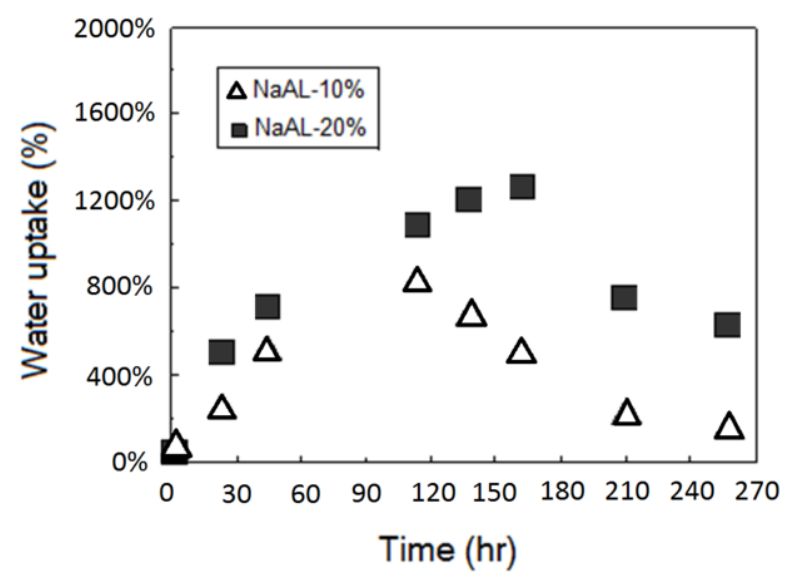

Fig. 4. Water uptakes of new designed drug-release system having a breathable polyurethane film-backed sodium alginate (NaAL) hydrocolloid acrylated adhesive layer.

Water uptakes of new designed drug-release systems having a breathable polyurethane film-backed carboxymethylcellulose (CMC) hydrocolloid acrylated adhesive layer are shown in Figure 2. Comparing the water absorption of components, carboxymethylcellulose (CMC) showed a relatively low capacity for water absorption. Low incorporation of CMC would show relatively low water uptake in resulting materials as shown in Figure 2. Even after 240 hours, the water uptake value was only at $80 \%$ (Figure 2). Relatively low water uptake in designed drug-release systems containing hydrocolloid components was observed in the corresponding low incorporation of hydrocolloid components. Low incorporation of CMC showed corresponding low water uptake (Figure 2).

Similarly, low incorporation of carbomer U10 showed corresponding low water uptake (Figure 3). Carbomer is a polymeric mixture of acrylic acid cross-linked with a polyfunctional compound, hence, a poly (acrylic acid) or polyacrylate. In general, carbomer was employed as a suspending agent for pharmaceuticals and drug-release systems. Incorporation of carbomer U10 would be important for the clinical application of drug-release systems. In this case, relatively high incorporation of carbomer U10 showed a corresponding high water uptake value. The designed drug-release system having breathable polyurethane film-backed carbomer U10 hydrocolloid acrylated adhesive layer could be a potential candidate for moisture healing and wound management [1]. Also, the carbomer U10 infused materials could provide a good platform for incorporation of hydrophilic drug systems. The remarkably high water uptake value of $4000 \%$ was observed in the sample U10-30\% as shown in Figure 3.

Sodium alginate $(\mathrm{NaAL})$ is a natural polymeric material with an excellent ability for water absorption. Also, sodium alginate (NaAL) could be employed as a suspending agent for pharmaceuticals and drug-release systems. The incorporation of sodium alginate (NaAL) is important for the clinical application of drug-release systems. Similarly, low incorporation of sodium alginate (NaAL) showed corresponding low water uptake during the allotted time (Figure 4). Water uptake values of drug-release systems having the hydrocolloid component of $10 \%$ sodium alginate(NaAL) increased with time (up to 120 hours); however, decreasing water uptake values of drug-release systems having the hydrocolloid component of $10 \%$ sodium alginate (NaAL) were observed after 120 hours. The water uptake value of drug-release systems having $10 \%$ sodium alginate (NaAL) was observed at $800 \%$ (Figure 4). Similarly, decreasing water uptake values of drug-release systems having the hydrocolloid component of $20 \%$ sodium alginate (NaAL) were observed after 160 hours. The water uptake of drug-release system having the hydrocolloid component of $20 \%$ sodium alginate $(\mathrm{NaAL})$ was observed at the value of 
$1200 \%$. The relatively low water uptake of drug-release systems having breathable polyurethane filmbacked $20 \%$ sodium alginate hydrocolloid acrylated adhesive layer was observed at the value of $600 \%$ after 240 hours. The water uptake value of $600 \%$ for 240 hours is almost half the uptake value of $1200 \%$ for 160 hours. This might be due to the dissociation of sodium alginate in the designed drugrelease system having breathable polyurethane film-backed sodium alginate (NaAL) hydrocolloid acrylated adhesive layer [26-28]. The interpenetrating polymer network (IPN) structure of the designed drug-release system would break down. Comparing incorporation of carbomer U10 with sodium alginate $(\mathrm{NaAL})$, drug-release systems containing carbomer with a crosslinked interpenetrating polymer network (IPN) would provide a relatively stronger structure than the drug-release systems containing sodium alginate (NaAL). The interpenetrating polymer network (IPN) structure of the designed drug-release system with carbomer U10 would not break down in the application of wound management. Even if the structure breaks down after a period of time, however, it could be a potential candidate the clinical application of drug-release.

\section{Conclusion}

New combined materials with a breathable PU film-backed hydrocolloid acrylated adhesive layer were designed and prepared for the clinical application of wound management and drug-release systems. All drug-release systems having a polyurethane film-backed hydrocolloid acrylated adhesive layer showed greater water uptakes at increasingly rapid rates during fixed time periods. After 114 hours, water uptake rates of drug-release systems with $20 \%$ hydrocolloid components measured $160 \%$, $1100 \%$, and $1870 \%$ greater when combined with $\mathrm{CMC}, \mathrm{NaAL}$ and carbomer, respectively. When comparing the incorporation of carbomer with $\mathrm{NaAL}$, drug-release systems containing carbomer with a crosslinked interpenetrating polymer network (IPN) yielded a stronger structure relative to the drugrelease systems containing NaAL. The IPN structure of the designed drug-release system with carbomer did not break down during wound management applications. Further, in spite of some structural break down after a fixed time, this could be a potential candidate in the clinical application of drugrelease systems. Furthermore, drugs could be incorporated into the adhesive layer via the incorporation of hydrocolloid components.

\section{Acknowledgement}

The authors would like to thank the Chang Gung Memorial Hospital Grant (CMRPG290471) and the Fu Jen Catholic University Grant (410031044033) for financially supporting this research. The authors also acknowledge for Ms. S.H. Lai for her technical assistance.

\section{References}

[1] E.A. O'Toole and J.E. Mellerio, "Wound Healing” Rook's Textbook of Dermatology, 8th ed., Oxford: Wiley- Blackwell Publication, New York, 2010, pp. 1-27.

[2] D.J. Liaw, C.C. Huang and B.Y. Liaw, Synthesis and properties of polyurethanes based on bisphenol-S derivatives, Polymer 39 (1998), 3529-3535.

[3] D.J. Liaw, C.C. Huang, H.C. Sang and E.T. Kang, Intramolecular hydrophobic aggregation of amphiphilic polysulfobetaine with various hydrophobic groups in aqueous solution, Langmuir 15 (1999), 5204-5211. 
[4] S. Ito, M. D.J. Liaw, T.P. Chen and C.C. Huang, Novel active ester-bridged copolynorbornene materials containing terminal functional hydroxyl, amino, methacryloyl or ammonium groups via ring-opening metathesis polymerization, J. Polym. Sci., Part A: Polym. Chem. 43 (2005), 4233-4247.

[5] D.J. Liaw and C.C. Huang, Synthesis and characterization of the poly (sulfobetaine)s and the corresponding cationic polymers derived from N,N'-dialkylamino phenyl methacrylamide, Macromol. Chem. Phys. 201 (2000), 1101-1107.

[6] D.J. Liaw, T.P. Chen and C.C. Huang, Self-assembly aggregation of highly stable copolynorbornenes with amphiphilic architecture via ring-opening metathesis polymerization, Macromolecules 38 (2005), 3533-3538.

[7] D.J. Liaw, C.C. Huang and S.M. Hong, Novel doubly polymerizable functional norbornene: Synthesis, reactivity and its macromolecular architectures from dual cure via ring-opening metathesis polymerization and radical photopolymerization, J. Polym. Sci., Part A: Polym. Chem. 44 (2006), 6287-6298.

[8] G. Zhai, S.C. Toh, W.L. Tan, E.T. Kang, K.G. Neoh, C.C. Huang and D.J. Liaw, Poly (vinylidene fluoride) with grafted zwitterionic polymer side chains for electrolyte-responsive microfiltration membranes, Langmuir 19 (2003), $7030-7037$.

[9] D.J. Liaw, C.C. Huang and W.H. Chen, Color lightness and highly organosoluble polyamides, polyimides and poly(amide-imide)s based on noncoplanar 2,2'-dimethyl-4,4'-biphenylene units, Polymer 47 (2006), 2337-2348.

[10] D.J. Liaw, C.C. Huang and E.T. Kang, Novel fluorescent polynorbornenes with multi-functional armed structure by using highly stable block macroinitiators via a combination of living ring-opening metathesis polymerization and atom transfer radical polymerization, Polymer 37 (2006), 3057-3064.

[11] D.J. Liaw, C.C. Huang, S.M. Hong, W.H. Chen, K.R. Lee and J.Y. Lai, Molecular Architecture effect on active structure of polynorbornenes with pendant $\alpha, \beta$-unsaturated amide or ester bridged chains via ring-opening metathesis polymerization, Polymer 47 (2006), 4613-4621.

[12] T. Katsumata, M. Maitani, C.C. Huang, M. Shiotsuki and T. Masuda, Synthesis and properties of various poly (diphenylacetylenes) containing tert-amine moieties, Polymer 49 (2008), 2808-2816.

[13] B.W. Hashimotob, N. Svizero, R.M. Carvalho, C. Yiu, F.A. Rueggeberg, S. Foulger, T. Saito, Y. Nishitanii, M. Yoshiyamai, F.R. Tay, D.H. Pashley, F.R. Tay and D.H. Pashley, Effects of resin hydrophilicity on water sorption and changes in modulus of elasticity, Biomaterials 26 (2005), 6449-6459.

[14] C.C. Huang and G.W. Chang, Designed nerve conduits derived from chitosan-coated alginate layers, PMSE Preprints Am. Chem. Soc. Div. Polym. Mater. Sci. Eng. 101 (2009), 465-466.

[15] D.J. Liaw, C.C. Huang and E.T. Kang, Effect of architecture and environments on polymeric molecular assemblies of novel amphiphilic diblock copolynorbornenes with narrow polydispersity via living ring-opening metathesis polymerization (ROMP), J. Polym. Sci., Part A: Polym. Chem. 44 (2006), 2901-2911.

[16] C. Silve, E. Lopez, B. Vidal and D.C. Smith, Nacre initiates biomineralization by human osteoblasts maintained in vitro. Calcif. Tissue Int. 51 (1992), 363-369.

[17] G. Atlan, O. Delatre, S. Berland, A. Le Faou, G. Nabias, D. Cot and E. Lopez, Interface between bone and nacre implants in sheep, Biomaterals 20 (1997), 1017-1022.

[18] J.E. Barralet, L. Grover, T. Gaunt, A.J. Wright and I.R. Gibson, Preparation of macroporous calcium phosphate cement tissue engineering scaffold, Biomaterials 23 (2002), 3063-3072.

[19] J.M. Karp, M.S. Shoichet and J.E. Davies, Bone formation on two dimensional poly (DL-lactide-co-glycolide) (PLGA) films and three dimensional PLG Shoichet, A tissue engineering scaffolds in vitro, J. Biomed. Mater. Res. A 64 (2003), 388-396.

[20] J.A. Roether, J.E. Gough, A.R. Boccaccini, L.L. Hench, V. Maquet and R. Jerome, Novel bioresorbable and bioactive composites based on bioactive glass and polylactide foams for bone tissue engineering, J. Mater. Sci. Mater. Med. 13 (2002), 1207-1214.

[21] G. Zhai, S.C. Toh, W.L. Tan, E.T. Kang, K.G. Neoh, C.C. Huang and D.J. Liaw, Poly (vinylidene fluo-ride) with grafted zwitterionic polymer side chains for electrolyte-responsive microfiltration membranes, Langmuir 19 (2003), 7030-7037.

[22] E.T. Kang, K.G. Neoh, W. Chen, K.L. Tan, D.J. Liaw and C.C. Huang, Surface structures and adhesion characteristics of poly (tetrafluoroethylene) films after modification by graft copolymerization, J. Adhesion Sci. Technol. 10 (1996), 725-743.

[23] Z.F. Li, E.T. Kang, K.G. Neoh, K.L. Tan, C.C. Huang and D.J. Liaw, Surface structures and adhesive-free adhesion characteristics of polyaniline films after modification by graft copolymerization, Macromolecules 30 (1997), 33543362.

[24] W. Chen, K.G. Neoh, E.T. Kang, K.L. Tan, D.J. Liaw and C.C. Huang, Surface modification and adhesion characteristics of polycarbonate films after graft copolymerization, J. Polym. Sci., Part A: Polym. Chem. 36 (1998), $357-$ 366. 
[25] H.C. Korting, C. Schöllmann and R.J. White, Management of minor acute cutaneous wounds: Importance of wound healing in a moist environment, Journal of the European Academy of Dermatology and Venereology 25 (2011), $130-137$.

[26] K.F. Cutting, Managing wound exudate using a super-absorbent polymer dressing: A 53-patient clinical evaluation, Journal of Wound Care 18 (2009), 200-205.

[27] W. Wigger-Albert, M. Kuhlmann, D. Wilhelm, U. Mrowietz, K. Eichhorn, J. Ortega, A. Bredehorst and K-P. Wilhelm, Efficacy of a polyurethane dressing versus a soft silicone sheet on hypertrophic scars, Journal of Wound Care 18 (2009), 208-214.

[28] F. Rossi, X. Chatzistavrou, G. Perale and A.R. Boccaccini, Synthesis and degradation of agar-carbomer based hydrogels for tissue engineering applications, Journal of Applied Polymer Science 123 (2012), 398-408. 